\title{
Photobrightening in Lead Halide Perovskites: Observations, Mechanisms and Future Potential
}

Zahra Andaji-Garmaroudi, Miguel Anaya, Andrew J. Pearson, Samuel D. Stranks*

Cavendish Laboratory, Department of Physics, University of Cambridge, JJ Thomson Avenue, Cambridge CB3 0HE, UK

*sds65@cam.ac.uk

\section{Keywords: perovskite, photobrightening, recombination, luminescence, passivation}

There has been a meteoric rise in commercial potential of lead halide perovskite optoelectronic devices since photovoltaic cells (2009) and light emitting diodes (2014) based on these materials were first demonstrated. One key challenge common to each of these optoelectronic devices is the need to suppress non-radiative recombination, a process that limits the maximum achievable efficiency in photovoltaic cells and light emitting diodes. In this Progress Report, we dissect recent studies that seek to minimise this loss pathway in perovskites through a photobrightening effect, whereby the luminescence efficiency is enhanced through a light illumination passivation treatment. We highlight the sensitivity of this effect to experimental considerations such as atmosphere, photon energy, photon dose, and also the role of perovskite composition and morphology; under certain conditions there can even be photodarkening effects. Consideration of these factors is critical to resolve seemingly conflicting literature reports. We scrutinise proposed mechanisms, concluding that there is some consensus but further work is needed to identify the specific defects being passivated and elucidate universal mechanisms. Finally, we discuss the prospects for these treatments to minimise halide migration and push the properties of polycrystalline films towards those of their single-crystal counterparts. 


\section{Introduction}

Over the past decade, hybrid perovskites have re-energised interest in high-performance, lowcost devices for energy harvesting and light emission thanks to their remarkably clean semiconductor properties and solution processability. ${ }^{[1]}$ The range of possible compositions is an additional attractive feature of perovskites. Facile composition engineering enables the development of perovskite solar cells (PSCs) with band gaps tuned for maximum power conversion efficiency (PCE) in both single junction and tandem solar cell configurations, ${ }^{[2-4]}$ and perovskite light emitting diodes (PeLEDs) that can be designed to emit at energies across the visible and near-IR regions of the electromagnetic spectrum. ${ }^{[5]}$ Among the lead halide perovskites, methylammonium (MA)-based perovskites such as $\mathrm{CH}_{3} \mathrm{NH}_{3} \mathrm{PbI}_{3}\left(\mathrm{MAPbI}_{3}\right)$ are the most studied compositions, though mixed cation and/or halide compositions using Cesium (Cs), Formamidinium (FA) or bromide are attracting increasing attention due to their improved optical and electrical properties. ${ }^{[2,6-11]}$ For PSCs, the state-of-the-art PCE already stands at $25.2 \%$ despite their short development timeframe, ${ }^{[12]}$ with this headline figure-of-merit efficiency surpassing that reached by several alternative classes of photovoltaics, both emerging (e.g., organic semiconductors, dye-sensitized and quantum dot configurations) and established (e.g., CdTe, CIGS and polycrystalline Si). ${ }^{[13]}$

Whilst the efficiencies of PSCs $(>25 \%)$ and PeLEDs $(>20 \%)^{[14]}$ are already at levels that are viable for technology transfer, from a research standpoint the challenge remains to make these technologies operate as efficiently and stably as possible. ${ }^{[15,16]}$ The necessary transition requires optimisation of several factors fundamental to device performance, for example, optical management (the in- and out-coupling of light), ${ }^{[15]}$ defect control, ${ }^{[3]}$ and charge carrier transport, extraction and recombination. ${ }^{[17]}$ The latter can be defined according to whether the recombination process results in the emission of a photon (radiative recombination) or phonon(s) (non-radiative recombination), with suppressed non-radiative recombination being 
a prerequisite for maximum device efficiency following the reciprocity relationships in the Shockley-Queisser efficiency formulations. ${ }^{[18-20]}$ Non-radiative recombination introduces a loss to the open-circuit voltage $\left(\mathrm{V}_{\mathrm{OC}}\right)$ of a solar cell device, where the $\mathrm{V}_{\mathrm{OC}}$ is a useful metric against which the quality of different solar cells, relative to their bandgaps, can be compared ${ }^{[20-22]}$. PSCs have been reported with non-radiative voltage loss values as low as $40 \mathrm{mV}$ and external device electroluminescence efficiencies of 9\%. ${ }^{[23,24]}$ Whilst these figures are outstanding for the technology, there is still ample room for improvement particularly across the entire family of materials to truly enable their potential diverse device applications. Minimising non-radiative voltage losses will require consideration of non-radiative recombination pathways both within the bulk of the solar cell active layer and at the charge collection layer interfaces. ${ }^{[25,26]}$

The active layers in PSCs and LEDs are generally poly-crystalline films, which have a sizable density of sub-gap trap states $\left(\sim 10^{15} \mathrm{~cm}^{-3}\right)$ that act as non-radiative recombination centres. ${ }^{[27-}$ ${ }^{29]}$ Perovskite single-crystals nominally have superior optoelectronic properties compared to their poly-crystalline thin film analogues, with reported trap densities in single-crystals of $\sim 10^{10}-10^{12} \mathrm{~cm}^{-3} \cdot{ }^{[30,31]}$ Although a proof of concept has been very recently shown by Chen et al., ${ }^{[32]}$ the processing of single-crystals into thin film diode structures poses significant technical challenges especially for up-scaling, and these devices are still far below the performance of poly-crystalline counterparts. There is therefore the tantalizing prospect of exploiting the facile processibility of poly-crystalline films in a variety of device structures while attaining optoelectronic properties (cf. low defect densities) of single-crystals. To do so, one must investigate suitable defect passivation treatments for polycrystalline films that could be applied at several stages during device fabrication. An ideal treatment would target the interiors of individual grains as well as grain boundaries and interfaces to suppress all nonradiative recombination and overcome any charge-carrier transport limitations. In doing so, 
they would also inhibit ion migration processes that can lead to device operational instability. ${ }^{[22]}$ Such treatments may allow polycrystalline PSCs and related devices to be commercialised at ultra-high performance levels.

One promising route to reduce non-radiative recombination and improve charge transport in perovskite films is a light soaking treatment in the presence of oxygen and/or moisture. ${ }^{[33-35]}$ This passivation approach has many advantages including easy in-situ application and potential applicability to a range of perovskite compositions. As reported by our group and others, this process, hereafter referred to as photobrightening, can substantially enhance luminescence yields of polycrystalline thin films, ${ }^{[35]}$ reaching extremely low trap densities with corresponding improvements in PSC performance. However, atmospheric molecules such as oxygen and water (and their derivatives) are also known to degrade perovskite films particularly over extended exposure periods. ${ }^{[33,36,37]}$ Therefore, the question over the universality of the effect with different systems and conditions and long-term stability issues could limit the commercial viability of this treatment. In this Progress Report, we summarise key results in the use of light to boost perovskite optoelectronic quality, as well as dissect the current understanding of the underlying mechanisms. From this, we consider how experimental factors, namely atmosphere, photon dose and photon energy, affect perovskites with different compositions and morphologies in order to comment on the universal applicability of this treatment. The opportunities are discussed alongside the limitations of such an approach, and we highlight how we could mimic the positive effects of oxygen and light using alternative strategies that may not lead to long-term stability issues. This work therefore presents a framework that will direct future research on defect passivation approaches and our fundamental understanding of carrier recombination in perovskites. 


\section{Observations of photobrightening and photodarkening effects}

Research into the photobrightening effect has been primarily motivated by the need to understand the fundamental mechanisms that govern perovskite light emission. The wide range of photoluminescence (PL) lifetimes and quantum efficiencies reported by different groups for the same nominal perovskite compositions, e.g. $\mathrm{MAPbI}_{3}$, has also exacerbated the need to decouple intrinsic and extrinsic effects. In 2014, Stranks et al. reported comparable kinetics between the increase in PL intensity from a $\mathrm{MAPbI}_{3}$ film during extended photoexcitation in ambient laboratory air conditions, and the concomitant increase in open-circuit voltage of a $\mathrm{MAPbI}_{3}$ PSC device during illumination (Figure 1a). ${ }^{[27]}$ Corresponding enhancements have also been determined for PL lifetimes under different atmospheres, ${ }^{[38,39]}$ indicating that the photobrightening effect impacts upon the relative fraction of (non-) radiative decay pathways within the samples. Nevertheless, others have reported 'photodarkening' processes in nominally similar structures and even seemingly comparable experimental conditions. ${ }^{[38,40]}$ For example, Gottesman et al. showed that a $\mathrm{MAPbI}_{3}$ film (encapsulated and thus protected from the environment) subjected to white light illumination undergoes a reversible decrease in PL intensity over time (Figure $1 \mathrm{~b}$ ), ${ }^{[40]}$ with the recovery period taking place only in dark conditions. Both photobrightening and photodarkening effects have been widely reported in the literature; a summary of the observations reported in a selection of studies we discuss in this report are shown in Table 1, alongside key experimental parameters, highlighting the seemingly conflicting behaviours described. This apparent contradiction will be discussed in subsequent sections by considering a myriad of factors that influence the photobrightening processes, where the interplay between mobile ions, external molecular species and the perovskite film morphology complicates efforts to universally predict the extent of any PL change and its magnitude. We note here that the PL lifetime, ascertained from some characteristic decay of the PL signal over time after excitation (which is in general non- 
exponential), depends strongly on the recombination regime and therefore excitation fluence. ${ }^{[41,42]}$ Therefore, care is needed in the extraction and interpretation of this parameter, and any value must be reported with reference to the excitation conditions. In this work, we report PL lifetimes from measurements in a 'trap-limited' recombination regime in which increases or decreases in this parameter qualitatively correspond to increases or decreases in luminescence yields.
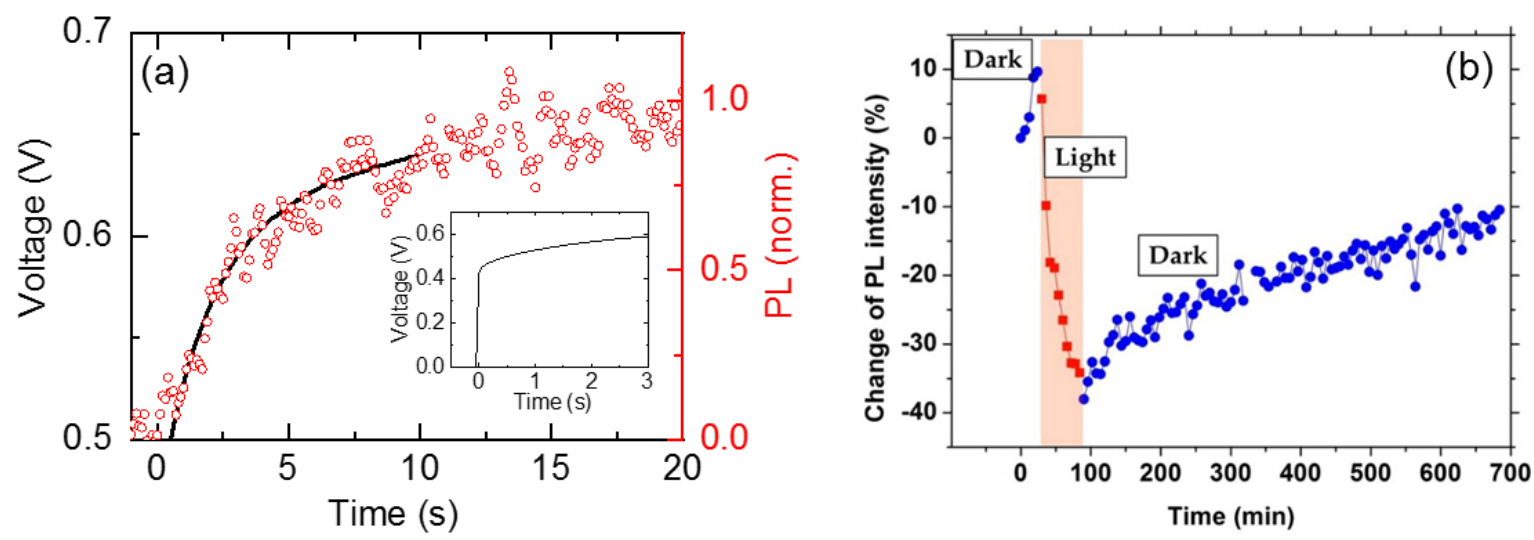

Figure 1: Summary of data from two reports on photobrightening and photodarkening. (a) Open-circuit voltage (black data) rise following illumination with a white-light LED source with intensity creating an equivalent number of excitations to 1-sun illumination, and PL intensity rise (red data) over time for a film photoexcited with a laser source (532-nm continuous wave (CW) laser, approximately $60 \mathrm{~mW} / \mathrm{cm}^{2}$, equivalent to $\left.1 \mathrm{sun}\right)$. Measurements were performed in ambient laboratory air. Reproduced with permission. ${ }^{[27]}$ Copyright 2014 , American Physical Society. (b) PL changes over time in a dark-light-dark cycle for an encapsulated $\mathrm{MAPbI}_{3}$ film, showing reversible decrease in PL upon light illumination with a white light source with intensity of $<10 \mathrm{~mW} / \mathrm{cm}^{2}$ ( 0.1 sun). Reproduced with permission. ${ }^{[40]}$ Copyright 2015, American Chemical Society (ACS). Further permissions related to the material excerpted should be directed to ACS. 
Table 1: Overview of reports on light-induced changes in perovskite PL intensity.

\begin{tabular}{|c|c|c|c|c|c|c|c|}
\hline Perovskite & Environment & $\begin{array}{l}\text { Excitation } \\
\text { wavelength } \\
\text { (nm) }\end{array}$ & 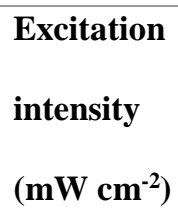 & $\begin{array}{l}\text { Light } \\
\text { soaking } \\
\text { time (s) }\end{array}$ & $\begin{array}{l}\text { Photon } \\
\text { dose } \\
\left(\mathrm{J} \mathrm{cm}^{-2}\right)\end{array}$ & $\begin{array}{l}\text { Photo- } \\
\text { brightening } \\
\text { or darkening }\end{array}$ & Ref \\
\hline $\mathrm{MAPbI}_{3}$ & $\begin{array}{l}\text { Depleted } \mathrm{O}_{2} \\
\text { Atmosphere }\end{array}$ & 507 & 300 & 800 & 240 & Brightening & {$[38]$} \\
\hline $\mathrm{FAMACsPbI}_{3}$ & $\begin{array}{l}\text { Vacuum }\left(10^{-5}\right. \\
\left.-10^{-6} \text { mbar }\right)\end{array}$ & 425 & 200 & 50 & 10 & Brightening & [43] \\
\hline $\mathbf{M A P b I}_{3}$ & $\begin{array}{l}\text { Vacuum (not } \\
\text { stated) }\end{array}$ & 507 & 300 & 587 & 180 & Brightening & [44] \\
\hline $\mathbf{M A P b I}_{3}$ & $\mathrm{~N}_{2}$ & 500 & 300 & 900 & 270 & Brightening & {$[33]$} \\
\hline $\mathbf{M A P b I}_{3}$ & $\mathrm{~N}_{2}$ & 532 & 150 & 100 & 15 & Brightening & [35] \\
\hline MAPbI3 & $\mathrm{O}_{2}$ & 500 & 300 & 900 & 270 & Brightening & [33] \\
\hline $\mathbf{M A P b I}_{3}$ & $\mathrm{O}_{2}$ & 514 & 1500 & 100 & 150 & Brightening & [34] \\
\hline $\mathrm{MAPbI}_{3}$ & $\mathrm{O} 2$ & 640 & 20 & 2400 & 48 & Brightening & [45] \\
\hline $\mathrm{MAPbI}_{3}$ & $\mathrm{O}_{2}$ & 532 & 150 & 300 & 450 & Brightening & {$[35]$} \\
\hline $\mathrm{MAPbI}_{3}$ & Humid Air & 532 & 150 & 600 & 90 & Brightening & [35] \\
\hline $\mathrm{MAPbBr}_{3}$ & $\mathrm{O}_{2}$ & 450 & 6.9 & 3000 & 21 & $\begin{array}{l}\text { First } \\
\text { brightening, } \\
\text { then } \\
\text { darkening }\end{array}$ & [36] \\
\hline $\mathrm{MAPbI}_{3}$ & Ambient & 532 & $\sim 60$ & 20 & 1.2 & Brightening & [27] \\
\hline $\mathrm{MAPbI}_{3}$ & Humid Air & 532 & 150 & 600 & 90 & Brightening & [35] \\
\hline $\mathrm{MAPbBr}_{3}$ & $\begin{array}{l}\text { Ambient, } \mathrm{O}_{2}, \\
\text { humid } \mathrm{N}_{2}\end{array}$ & 400 & 994 & 1500 & 1500 & Brightening & {$[46]$} \\
\hline $\mathrm{MAPbI}_{3}$ & $\begin{array}{l}\text { Humid } \mathrm{N}_{2} \text {, Air, } \\
\text { Humid Air }\end{array}$ & 405 & 500 & 300 & 150 & Brightening & [47] \\
\hline $\mathrm{MAPbI}_{3}$ & ambient & 532 & 400 & 55 & 22 & Brightening & {$[48]$} \\
\hline MAPbBr3 & Vacuum & 400 & 994 & 1500 & 1500 & Stable & [46] \\
\hline
\end{tabular}




\begin{tabular}{|l|l|l|l|l|l|l|l|}
\hline $\mathbf{F A P b}(\mathbf{I} / \mathbf{B r})_{3}$ & Vacuum & 400 & 15 & 70800 & 1100 & Stable & {$[49]$} \\
\hline MAPbBr 3 & $\mathrm{~N}_{2}$ & 400 & 994 & 1500 & 1500 & Stable & {$[46]$} \\
\hline FAMACs & Ambient & 532 & 60 & 1800 & 108 & Stable & {$[50]$} \\
$\mathbf{P b ( I , B r )}$ & & & & & & & \\
\hline MAPbBr 3 & Ultra-high & 450 & 6.9 & 3000 & 21 & Darkening & {$[36]$} \\
\hline MAPbI 3 & Eacuum & & & & & & \\
\hline MAPbI3 & Encapsulated & White light & $<10$ & 3600 & 36 & Darkening & {$[40]$} \\
\hline MAPbI 3 & Air & 405 & 500 & 300 & 150 & Darkening & {$[47]$} \\
\hline MAPbI & ambient & 457 & 100 & 55 & 5.5 & Darkening & {$[48]$} \\
\hline
\end{tabular}

\section{Sensitivity to experimental conditions}

A number of reports have shown that the sign (i.e. photobrightening or photodarkening) and extent of the luminescence change strongly depends on the experimental conditions, which we summarise in Figure 2. Galisteo-López et al. and Tian et al. first showed that oxygen-rich environments are critical for the dramatic increases in the PL intensity of $\mathrm{MAPbI}_{3}$ films under continuous illumination. ${ }^{[33,34]}$ The sensitivity of the surface to environmental gases was highlighted by Fang et al., ${ }^{[46]}$ who showed that the PL of $\mathrm{MAPbBr}_{3}$ single-crystals drops dramatically over time under illumination in vacuo, but then a PL rise was seen upon subsequent exposure to air. Similar atmospheric-dependent behaviour in the PL intensity has been reported in $\mathrm{CsPbBr}_{3}{ }^{[51,52]}$ Water molecules have also been shown to contribute to similar photobrightening effects as oxygen, and this can even be synergistic in the presence of both oxygen and water molecules. ${ }^{[35]}$ Anaya et al. quantified the extreme sensitivity of the processes to oxygen: in ultra-high vacuum (UHV) $\left(10^{-8} \mathrm{mbar}\right)$, the PL decreased under illumination, whereas at pressures as low as 1.3 mbar, the photobrightening effects could already be activated (Figure 2a). ${ }^{[36]}$ These results suggest that the photobrightening effects under continuous 
illumination may require at least a trace quantity of oxygen and/or other molecules. We note that there is an ongoing competition between photobrightening and photodarkening processes during illumination of such films as atmospheric molecules such as oxygen and water and even light are known to degrade perovskite films when continuously exposed to these stressors. ${ }^{[33,36,37,53]}$ Galisteo-López et al. demonstrated that photobrightening in $\mathrm{MAPbI}_{3}$ in ambient air was followed by a subsequent drop of the PL after tens of seconds (depending on the incident light intensity), ${ }^{[33]}$ though this is partly reversible when exposed to dry nitrogen. This rise in PL followed by a drop over longer time periods under illumination has also been reported, albeit at a lower darkening rate, in $\mathrm{O}_{2}$ and $\mathrm{N}_{2}$ atmospheres (cf. Figure 2a). ${ }^{[33]}$

(a)

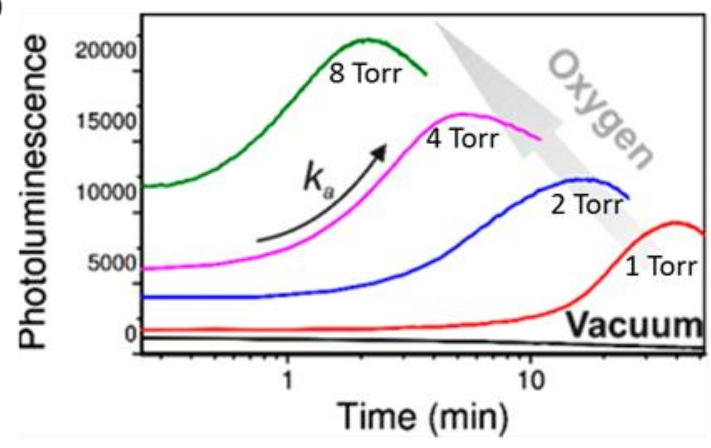

(c)

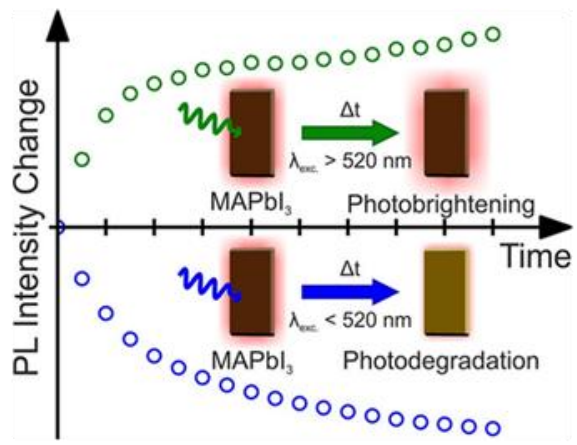

(b)

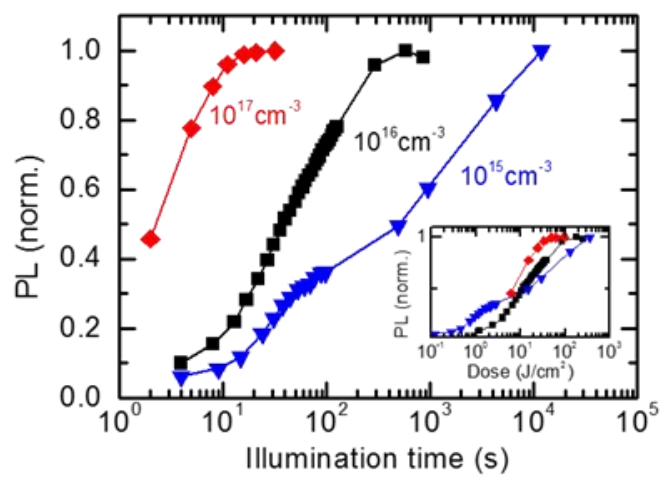

(d)

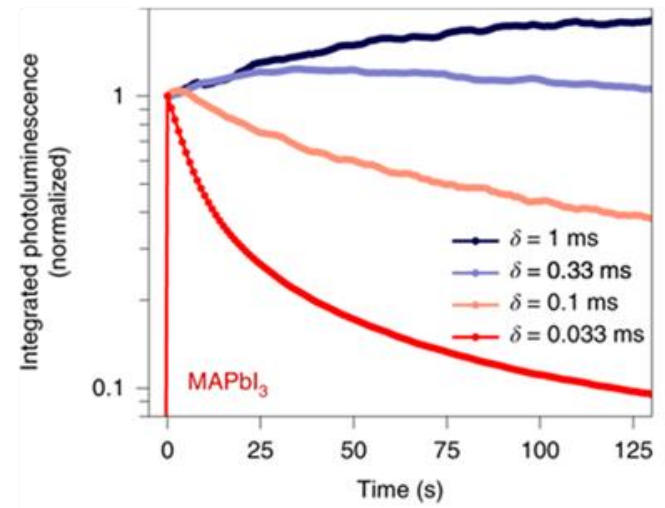

Figure 2: Summary of the impact of different experimental factors on photobrightening. (a) Light-induced PL changes over time for $\mathrm{MAPbBr}_{3}$ films exposed to different $\mathrm{O}_{2}$ pressures with 450-nm excitation $\left(6.9 \mathrm{~mW} / \mathrm{cm}^{2}\right)$. Reproduced with permission. ${ }^{[36]}$ Copyright 2018, ACS. (b) Normalized integrated PL from a $\mathrm{MAPbI}_{3}$ film over time under illumination at the stated 
excitation density with 507-nm pulsed excitation (1 MHz excitation rate). Inset shows the same data plotted with the total photon energy dose. Reproduced under the terms of the Creative Commons CC BY license ${ }^{[38]}$. Copyright 2019, Springer Nature. (c) The PL intensity change in a $\mathrm{MAPbI}_{3}$ film over time under illumination with either $457-\mathrm{nm}\left(100 \mathrm{~mW} / \mathrm{cm}^{2}\right)$ or $532-\mathrm{nm}(400$ $\mathrm{mW} / \mathrm{cm}^{2}$ ) laser excitation. Measurements were performed under ambient air conditions. Reproduced with permission. ${ }^{[48]}$ Copyright 2018, ACS. (d) Integrated PL intensity, normalized at time $\mathrm{t}=0$, from a $\mathrm{MAPbI}_{3}$ film held under vacuum (pressure $<10^{-5} \mathrm{mbar}$ ) with the stated times held in the dark $(\delta)$ between illumination with light pulses of duration $200 \mathrm{~ns}$ $\left(\sim 0.5 \mu \mathrm{J} / \mathrm{cm}^{2}\right.$ per pulse, $450-\mathrm{nm}$ excitation wavelength). Reproduced with permission. ${ }^{[54]}$ Copyright 2019, Springer Nature.

The photoexcitation conditions also play an important role in determining the extent of photobrightening. Galisteo-Lopez et al. ${ }^{[33]}$ found that photobrightening and photodarkening depend on the intensity of the excitation, and the rate of both processes increases with intensity. This suggests that the photon 'dose' is critical in these processes. deQuilettes et al. ${ }^{[38]}$ demonstrated this by illuminating with different excitation densities and monitoring the PL changes over time (Figure $2 b$ ). The time taken for the PL to rise and reach a stabilised emission output depends on the excitation density, where the time to reach the stabilised emission is longer at lower excitation densities. However, when considering the photon dose in each case (Figure $2 \mathrm{~b}$, inset) these curves all overlay, revealing a critical photon dose for stabilised emission in these $\mathrm{MAPbI}_{3}$ films under these atmospheric conditions to be $\sim 100 \mathrm{~J} / \mathrm{cm}^{2}$. We note that these measurements were performed with relatively moderate vacuum levels (pressure of $\sim 10^{-3}-10^{-4}$ mbar) and thus the trace oxygen present under such conditions leads to the observed net rise in PL, again highlighting the sensitivity of the effect to oxygen (cf. Figure 2a). We note that this sensitivity to oxygen can also be seen in measurements performed under nominal 
nitrogen conditions, which will ultimately still contain trace concentrations of oxygen species. Sutter-Fella et al. also showed that the transition from photobrightening to photodarkening is dependent on photon dose. ${ }^{[55]}$

The role of photon energy was studied by Quitsch et al. ${ }^{[48]}$. By light soaking $\mathrm{MAPbI}_{3}$ thin films for the same duration under ambient laboratory air conditions, they observed a photodarkening effect when photo-exciting with photons below a critical wavelength of $\sim 520$ $\mathrm{nm}$ and a photobrightening effect above this wavelength (Figure 2c). ${ }^{[48]}$ They proposed that this energy dependence of the PL involves the photoexcitation of residual $\mathrm{PbI}_{2}$, where the threshold of $\sim 520 \mathrm{~nm}$ corresponds to the band edge of $\mathrm{PbI}_{2}$, and this can trigger a decomposition process for $\mathrm{MAPbI}_{3}$. Merdasa et al. found that light soaking in ambient conditions could further exaggerate this energy-dependent effect as $\mathrm{PbI}_{2}$-rich regions on the surface grow over time under illumination. ${ }^{[56]}$ This leads to vastly different charge-carrier recombination processes through largely inefficient injection of charge carriers into the perovskite surface via a $\mathrm{PbI}_{2}$ intermediary when photo-exciting at low wavelengths.

Another crucial factor is not just how much photon dose arrives, but when this does arrives and whether there is a relaxation (recovery) time between the doses. Motti et al. recently studied the impact of the relaxation time between illumination on the photobrightening and -darkening effects in $\mathrm{MAPbI}_{3}$ and $\mathrm{MAPbBr}_{3}$ films under high vacuum conditions $\left(<10^{-5} \mathrm{mbar}\right.$, Figure $\left.2 \mathrm{~d}\right)$. They found that with a fixed excitation pulse width but increasing length of time between pulses, they could modulate from a photodarkening (relaxation times between pulses of 0.033 $\mathrm{ms}$ ) to a photobrightening (relaxation times of $1 \mathrm{~ms}$ ) effect over early times. This suggests that even in the nominal absence of oxygen a photobrightening effect can be observed provided there is sufficient relaxation time between pulses. This result speaks to the ongoing interplay between mechanisms leading to photobrightening and photodarkening even on short time scales. Nevertheless, we note that longer illumination times (and therefore higher effective 
photon doses) would be required to explore this interplay further, especially to validate whether there would be a net photodarkening process at longer exposure times.

Finally, the rate of photobrightening is strongly dependent on temperature. The phenomenon is slowed down substantially at lower temperatures, requiring an order of magnitude larger photon dose to reach a similar stabilised emission output at $190 \mathrm{~K}$ than at $300 \mathrm{~K} .^{[38]}$

\section{Sensitivity to Sample Morphology, Composition and Passivation}

The photobrightening effect is also dependent on the perovskite thin film morphology and composition. deQuilettes et $a l^{[38]}$ investigated how microstructure impacts the PL enhancement in poly-crystalline $\mathrm{MAPbI}_{3}$ films under illumination over time. By correlating scanning electron microscopy (SEM) micrographs and confocal PL microscopy images, they showed that light-soaking preferentially increases the luminescence from regions with higher trap state densities (dark spots in initial PL images) and also induces a redistribution in local emission intensities (Figure 3a and b). These observations hint that perovskite films of better initial quality exhibit more limited photo-induced changes. Similar observations were made by Brenes et $a l .{ }^{[47]}$ using in-situ micro-PL measurements under different atmospheric environments. Tian et al. showed that heterogeneity within the perovskite, such as morphological grain size variations, affects the extent to which photobrightening takes place ${ }^{[45]}$; they found that smaller grains exhibited faster PL enhancement than larger grains They also demonstrated that different local regions within the same perovskite film have vastly different PL enhancement transients (Figure 3c). ${ }^{[34]}$ Other works have reported spatially dependent PL blinking effects with random on-off fluctuations of non-radiative channels that are particularly exaggerated in nano-sized crystals ${ }^{[57-59]}$; this blinking effect is also strongly dependent on the surrounding atmosphere and temperature. ${ }^{[60]}$ 

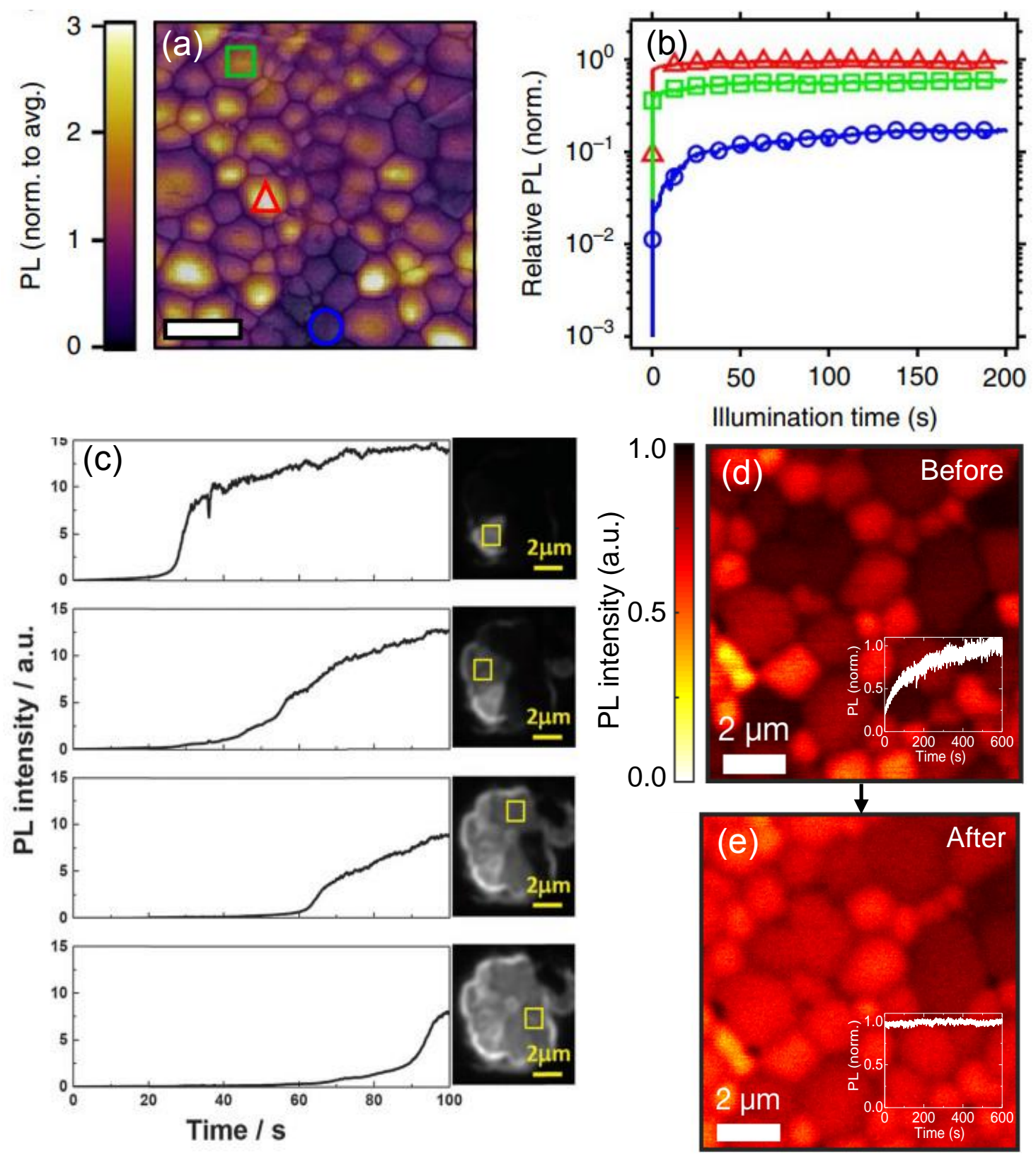

Figure 3. (a) Confocal PL map of a $\mathrm{MAPbI}_{3}$ film with semi-transparent SEM image overlaid, (scale bar $2 \mu \mathrm{m}) .^{[38]}$ (b) PL intensity of the bright (red), intermediate (green) and dark (blue) regions denoted in (a) over time under illumination (470-nm laser, $40 \mathrm{MHz}, \sim 0.03 \mu \mathrm{J} . \mathrm{cm}^{-2}$ per pulse). Reproduced under the terms of the Creative Commons CC BY license ${ }^{[38]}$. Copyright 2016, Springer Nature. (c) PL enhancement of different local domains (as highlighted) in a $\mathrm{MAPbI}_{3}$ film over time under illumination $\left(1500 \mathrm{~mW} / \mathrm{cm}^{2}, 514-\mathrm{nm} \mathrm{CW}\right.$ excitation $)$. Adapted with permission from the Royal Society of Chemistry (2015). Reproduced with permission. ${ }^{[34]}$ 
Copyright 2015, Royal Society of Chemistry. (d,e) Confocal PL maps of the same region of a $\mathrm{MAPbI}_{3}$ film (d) before and (e) after in-situ light soaking for 10 minutes under excitation with a 532-nm laser equivalent to $\sim 10$ sun under humid air (45\% relative humidity). Insets to (d) and (e): emission stability of the films in dry air before and after the treatment, respectively, under continuous illumination at 532-nm with intensity equivalent to $\sim 10$ sun. Reproduced under the terms of the Creative Commons CC BY license. ${ }^{[35]}$ Copyright 2017, Cell Press.

The ability to enhance the PL in both $\mathrm{MAPbI}_{3}$ and $\mathrm{MAPbBr}$ samples through light and atmospheric treatments motivates the important question of whether photobrightening is a general phenomenon in all perovskite compositions. In mixed halide systems such as $\mathrm{MAPb}(\mathrm{I} / \mathrm{Br})_{3}$, there is a photo-induced ionic segregation process that leads to a red-shifting of the PL emission to lower energies corresponding to iodide-rich sites. ${ }^{[61]}$ Since carriers can accumulate on these low-bandgap regions, fractions of radiative recombination can be very high, and thus there is a large overall increase in the PL intensity. ${ }^{[61-64]}$ We note that unintentional fractional deviations in the precursor solution stoichiometry can also impact the extent and sign of photobrightening (or darkening) in perovskite films, a variable which would further contribute to the inconsistencies of reported results. ${ }^{[65]}$ It is reported that the PL intensity increases under continuous illumination in oxygen-rich conditions even in multi-cation perovskites such as (FA,MA,Cs) $\mathrm{PbI}_{3}$, but the extent and rate of this increase is in general lower compared to the $\mathrm{MAPbI}_{3}$ analogues, suggesting there is at least to some degree a suppression of the photobrightening or photodarkening effects in those compositions. ${ }^{[43,62]}$ The mixedcation systems also in part retard the halide segregation processes,${ }^{[49,66]}{ }^{[7]}$ though we note that the degree of this inhibition depends on fabrication, crystallinity and experimental conditions. Generally, continuously illuminating mixed-cation, mixed-halide systems in oxygen-rich 
conditions leads to an overall passivation of deep traps, and a halide segregation process, with both processes contributing to a net increase in luminescence. ${ }^{[62]}$

In addition, these mixed-cation, mixed-halide systems have local spatial chemical heterogeneity leading to small local variations in the bandgap on the order of tens of millielectronvolts. This variation leads to shallow states and subsequently local accumulation of one type of photo-excited carrier through a photo-doping effect. ${ }^{[67]}$ Interestingly, independent of the atmosphere and changes in PL intensity, the influence of these shallow states can be reduced by light-soaking these systems, likely because the initial well inter-mixed halide distributions (leading to shallow states) are converted to distinctly segregated iodide and bromide regions. ${ }^{[50]}$ It has also been reported that the light-induced changes can be suppressed through surface passivation of films. Abdi-Jalebi et al. observed that the PL intensity and wavelength in potassium-passivated (FA,MA, $\mathrm{Cs}) \mathrm{Pb}(\mathrm{I} / \mathrm{Br})_{3}$ films are more stable under illumination compared to unpassivated films, suggesting that the photoinduced migration processes are substantially inhibited in this composition even in ambient air conditions. ${ }^{[9]}$ This highlights that photoinduced changes are most prevalent in materials of lower starting quality (for example unpassivated films), whereas in high quality materials (e.g. passivated films) this effect will be drastically reduced. The impact of passivation is strikingly demonstrated when considering the $\mathrm{PL}$ of $\mathrm{MAPbI}_{3}$ films passivated through a light treatment in humid air, which homogenises and increases the local PL intensity distribution when comparing PL maps before (Figure 3d) and after (Figure 3e) the treatment. During the illumination treatment, there is a pronounced photobrightening effect (Fig. 3d, inset). However, once the sample reaches a stabilised emission level and the continuous illumination and humidity components are removed, further illumination shows that the luminescence is stabilised at enhanced levels with respect to the untreated film and no longer changes over time (Fig. 3e, inset). 
From our collective analysis above, we conclude that photobrightening is a general property of lead halide perovskites but the extent and rate of the effect depends on the experimental conditions (photon dose, photon energy, relaxation time, environment, temperature) and the sample properties (composition, morphology and degree of passivation). The apparent contradictions in the literature stem from the high sensitivity of the processes to these factors. We note that further studies on phase-stable $\mathrm{CsPbX}_{3}$ embodiments are needed to elucidate any mechanistic differences in the inorganic lead halide perovskite family.

\section{Mechanistic Insights}

As discussed in the previous sections, the photobrightening effect is sensitive to a myriad of factors, which makes it a very complicated phenomenon to understand. Nevertheless, the sensitivity to the conditions detailed above also provide different levers to adjust the processes, and therefore allow us to extract mechanistic insights to rationalise the observed behaviour.

The slow rise and/or drop of the PL intensity during light treatment (minutes) in various environments under typical solar excitation charge densities suggest that electronic charge transport and recombination processes cannot exclusively be responsible for the photobrightening effect. For example, if we simply consider the interplay between trap filling (100-ns time scale) and trap depopulation (100-ms time scale as a conservative slow estimate),

the system would reach equilibrium within a few hundreds of microseconds. ${ }^{[27,38,68]}$ Rather, such slow time scales are consistent with ionic (primarily halide) migration, oxygen diffusion, and/or chemical reactions on the surface involving halides and adsorption/ desorption of oxygen playing crucial contributing roles. deQuilettes et al. compared confocal PL maps with time-of-flight secondary-ion-mass-spectrometry (TOF-SIMS) data on the same scan region. They revealed that the 'photobrightened' region is deficient in iodide with respect to the non- 
illuminated surrounding region (Figure 4a), and that such a light-induced redistribution of halides depends on the photon dose. ${ }^{[38]}$ This is direct evidence for photo-induced ion migration, and the strong correlations between the extent of this effect and the observed photobrightening supports the idea that the mechanisms are intertwined. The hindering of the photobrightening effect in mixed cation systems, in which ion migration is significantly suppressed, ${ }^{[7,49,66]}$ is also consistent with the role of ions in the process; reduced defect densities in these mixed cation systems will also contribute to the suppressed effects, with both phenomena ultimately limiting the supply of reactant species and sites described below. The role of ions is also reflected in the strong temperature dependence of the photobrightening processes, with overall slower processes at lower temperatures consistent with slower ionic movement at low temperature. Activation energies for the photobrightening processes of $\sim 0.2 \mathrm{eV}$ are observed, which is broadly consistent with the activation energies for halide migration in $\mathrm{MAPbI}_{3}$ of $\sim 0.1-0.6$ $\mathrm{eV} .{ }^{[69,70]}$ Although the mechanism of ion migration in lead halide perovskites is still under debate, a plausible scenario relies on the ability of halides to migrate through halide defects in the perovskite structure. ${ }^{[54]}[61,69,70]$

Motti et al. proposed that the long-lived carrier traps associated with halide defects govern the photobrightening and photodarkening processes (even in the absence of oxygen) by inducing photo-chemical reactions of mobile ionic species at those sites. At low charge carrier excitation densities (longer excitation wavelengths), low repetition rates (1000 pulses/s) and low temperatures $(\sim 200 \mathrm{~K})$, they propose that photobrightening can take place in $\mathrm{MAPbI}_{3}$ through electron trapping at iodide interstitial defects and subsequent Frenkel pair trap annihilation (Figure 4b). ${ }^{[54]}$ This is also consistent with the recent mechanism proposed by Mosconi et.al. in which light illumination leads to the annihilation of Frenkel pairs. ${ }^{[44]}$ By contrast, lightinduced formation of defects in small volumes promoted by high repetition rates and high excitation densities (shorter wavelengths) has been linked to a bimolecular reaction in which 
two filled iodine traps could react to form an $\mathrm{I}_{2}$ molecule. Alternatively, and less probable, sequential electron and hole capture at an iodide interstitial defect pair can induce the formation of $I_{2}$. Either way, $I_{2}$ constitutes an electron trap that they propose gives rise to the photodarkening effect (Figure 4b). ${ }^{[54]}$

Other works have proposed mechanisms to understand the photobrightening effect related to atmospheric conditions. ${ }^{[33-35,47,53,71,72][73][36]}$ Illumination in general generates defects such as halide vacancies (for example through light-induced ion migration) or atomic lead species. ${ }^{[37,38]}$ It has been proposed that molecular oxygen adsorbs extremely well to perovskite surfaces and defect sites (e.g. the generated vacancies), and then photo-generated electrons can convert the oxygen to superoxide $\left(\mathrm{O}_{2}^{-}\right)$(Figure 4c). Thus, continuous exposure to light induces the formation of superoxide species at the perovskite surface, at the expense of bromine, carbon and nitrogen species (Figure 4d, phase I), a process that is partially reversible upon relaxation in the dark under ultra-high vacuum conditions (Figure 4d, phase II). ${ }^{[36]}$ The adsorption energy of such superoxide species is extremely high, on the order of chemi-sorption energies, particularly on neutral and negative halide vacancy sites $(\sim-3.90 \mathrm{eV}) \cdot{ }^{[47]}$ Calculations have shown that the $\mathrm{O}_{2}$ and $\mathrm{O}_{2}{ }^{-}$adsorption reduces the deep trap site density in $\mathrm{MAPbI}_{3} \cdot{ }^{\left[{ }^{[3]}\right.}$ Godding et al. proposed that the superoxide is a precursor in the formation of peroxide species (e.g. hydrogen peroxide), which in turn oxidises the atomic lead defects (traps) into benign PbO. ${ }^{[71]}$ They supported their mechanism by observing similar increases in luminescence yields and device performance by employing a direct post-treatment exposure of (FA, $\mathrm{Cs}) \mathrm{Pb}(\mathrm{I} / \mathrm{Br})_{3}$ films to hydrogen peroxide (i.e. without light). Such a mechanism could also explain the synergistic effects of oxygen and water, ${ }^{[35,47]}$ where moisture will assist the formation of hydrogen peroxide. In addition, moisture may instigate the partial decomposition of the perovskite at grain boundaries into passivating degradation products such as $\mathrm{PbI}_{2}$ and $\mathrm{PbO}$, thus converting a defective surface into a benign surface. ${ }^{[74-76]}$ Such thin surface layers may also supress inter- 
grain ion transport, which, along with the passivated defects, leads to the stabilisation of the emission after the sample is removed from the light and oxygen/humid atmosphere (cf. Figure $3 \mathrm{c}$ and $\mathrm{d})$. The crossover from photobrightening to photodarkening is due to a competitive process in which volatile organic products (i.e. MA and/or FA) are created via peroxide formation, and results in an irreversible darkening. ${ }^{[36,71]}$ The slow time scales involved are due to a combination of the interplay between the photobrightening and photodarkening processes, any slow diffusion processes (such as oxygen or ionic migration), and the specific atmospheric conditions limiting reactants.

A common requirement between the models proposed above is that there is a source of charge carriers (for example from photo-excitation or strong oxidising agents) to instigate photochemical reactions to ameliorate defect states. The reactants in these processes are atmospheric species and/or ionic species (halides, vacancies or interstitials). Nevertheless, there are still a number of questions remaining, and the proposed models do not yet agree on all aspects. One of the key outstanding questions relates to understanding the intrinsic nature of the defects that are being passivated or exaggerated through light-induced effects. A recent local structural and photophysics study revealed that the local sites associated with nonradiative decay are directly related to lattice strain. ${ }^{[77]}$ These strains are 'built-in' during the film growth and crystallization stages and may relate to grain boundaries. ${ }^{[78]}$ Other recent work suggests that light illumination in mild vacuum conditions can at least in part relieve this strain, resulting in enhanced PL properties and performance of photovoltaic devices. ${ }^{[43]}$ Such findings are consistent with recent reports that strain can impact performance and lead to operational instabilities; ${ }^{[79,80]}$ this is also consistent with the proposition that these non-radiative traps are in turn associated with ionic migration. Such strain formation and relief mechanisms and measurements will need to be reconciled with the atmospheric, photon dose and photon energy measurements to provide a universal picture for these effects. A complete picture to understand 
the dynamic interplay between ion migration, trap annihilation, and photoinduced chemical reactions in lead halide perovskites will require an in-depth understanding of the interrelated structural, chemical and photophysical properties across different length scales (macro down to nano-scale). Only through direct experimental evidence to understand these states will we be able to draw definitive conclusions on the associated photochemical reactions.

(a)
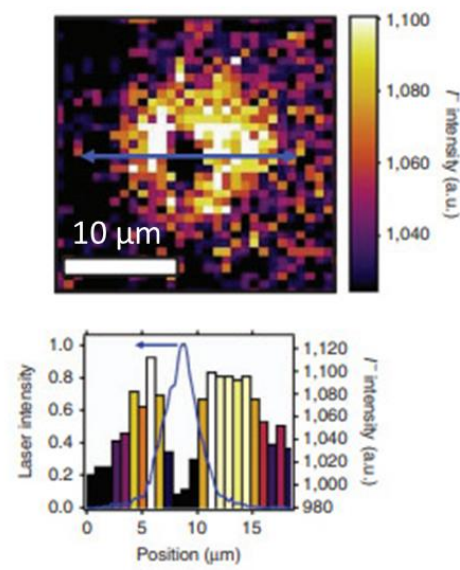

(b)

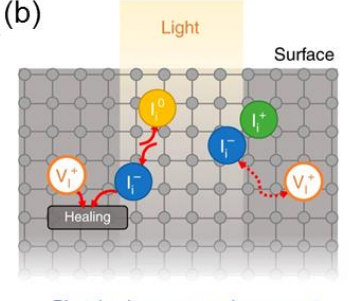

Photoluminescence enhancement

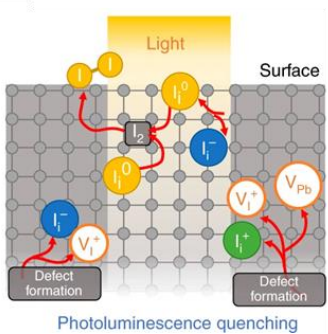

(c)

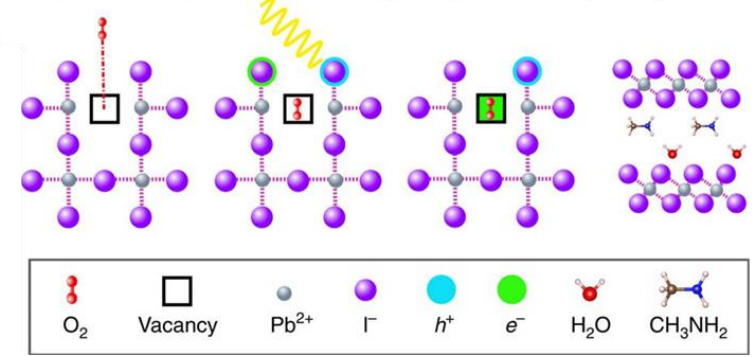

(d)

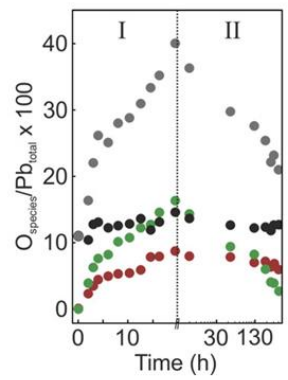

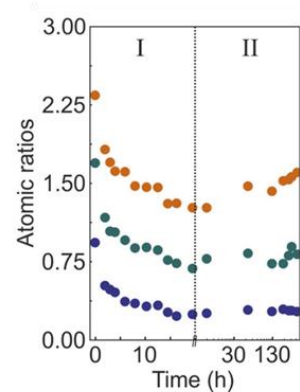

Figure 4. Summary figures from characterisation and mechanistic studies. (a) ToF-SIMS depth profiling image of the iodide distribution in $\mathrm{MAPbI}_{3}$ film after illumination in vacuo with a photon dose of $1.2 \mathrm{~kJ} / \mathrm{cm}^{2}$ (470-nm excitation). Reproduced under the terms of the Creative Commons CC BY license. ${ }^{[38]}$ Copyright 2016, Springer Nature. (b) Proposed photobrightening and photodarkening mechanisms in $\mathrm{MAPbI}_{3}$ films. The respective vacancies for each ion are noted by $V$, and interstitials with a subscript $i$. Reproduced with permission from Springer Nature (2019). ${ }^{[54]}$ (c) Mechanism of reaction steps of oxygen with $\mathrm{MAPbI}_{3}$. Reproduced under the terms of the Creative Commons CC BY license. ${ }^{[53]}$ Copyright 2017, Springer Nature. (d) $\mathrm{X}$-Ray Photoemission Spectroscopy analysis of a $\mathrm{MAPbBr}_{3}$ film upon photoexcitation under 
$\mathrm{O}_{2}$ exposure (phase I) and during recovery in dark under ultra-high vacuum conditions (phase II). Left panel shows the evolution of the oxygen (grey circles), water (black circles), superoxide (red circles), and oxide (green circles) signals with respect to $\mathrm{Pb}$, while the right panel shows the evolution of bromine (orange circles), carbon (dark green circles) and nitrogen species (blue circles). Reproduced with permission. ${ }^{[36]}$ Copyright 2018, ACS.

\section{How to maximally exploit these effects}

Although the underlying mechanisms of the photoinduced processes in perovskites are still under debate, the ability to enhance the properties of lead halide perovskites using such treatments under appropriate conditions is clearly established. We now ask how far we can improve the properties of polycrystalline films using such treatments. To do so, we must ensure the maximum photobrightening effects can be captured to achieve emissive and stable films while suppressing unwanted competing photodarkening or other photochemical processes which will dominate over longer term exposure. Thus, a practical strategy is to optimise the light and atmosphere exposure dose but then isolate the sample from these conditions and subsequently measure the 'treated' film and subsequently complete construction of any device comprising these films.

Brenes et al. showed that under optimised conditions of humid air ( 40\%-50\% relative humidity) and photon dose and energy $\left(10 \mathrm{~J} / \mathrm{cm}^{2}\right.$ with 532-nm excitation), they could increase the internal PL quantum efficiency values to over $80 \%$ from an initial value of $\sim 1 \%$ (Figure 5a), along with a more locally homogeneous emission distribution (cf. Figure $3 \mathrm{~d}$ and e). ${ }^{[35]}$ They observed this PL enhancement concomitant with an enhanced carrier lifetime to $>30 \mu \mathrm{s}$ under $\sim 1$ sun equivalent excitation conditions (compared to $6 \mu$ s before treatment) whilst maintaining carrier mobilities of $70-90 \mathrm{~cm}^{2} \mathrm{~V}^{-1} \mathrm{~s}^{-1}$ ascertained using time-resolved microwave conductivity measurements. These values are comparable to those obtained with the best 
chemical passivation approaches and single-crystal systems. ${ }^{[55,81-83]}$ Furthermore, the associated surface recombination velocity for holes in the treated film $(\sim 0.4 \mathrm{~cm} / \mathrm{s})$ is comparable to the best values reported for any semiconductor in $(0.15 \mathrm{~cm} / \mathrm{s}$ in $<111>$ crystalline silicon $^{[84,85]}$, which is remarkable considering that these are solution-processed polycrystalline films. PSCs constructed using the treated films as active layers exhibited higher PCEs (19.2\%) compared to control PSCs $(17.8 \%) .{ }^{[35]}$ The enhanced performance is also reflected in the faster rise to stabilised power output, and reaching higher values (Figure 5b), which is in turn consistent with the stabilised PL output (cf. Figure 3c and d) and reduced ionic migration in such treated configurations. ${ }^{[22,86][35]}$ We note that similar results were found by Godding et $a l .{ }^{[71]}$ in inverted device structures using films exposed to hydrogen peroxide, which is proposed to generate the same effect as photobrightening. Crucially, the photo-darkening processes in such passivated structures are also suppressed. Although this doesn't necessarily mean that ion migration is entirely inhibited, it may mean that any 'background' ion motion may not be detrimental for optoelectronic performance; though further long-term stability testing will be required to validate this assertion. Recently, Andaji-Garmaroudi et al. showed that light-soaking mixed-cation and mixed-halide films leads to the formation of an extremely thin and well-passivated iodide-rich surface layer on which charges accumulate and recombine with very high radiative efficiencies even at low excitation densities; the PL quantum efficiency of the film increases from $3 \%$ to over $45 \%$ (Figure $5 \mathrm{c}$ ). ${ }^{[62]}$ These promising demonstrations show the potential for such passivation strategies to enable new device structures and push perovskite semiconductors towards their limits. Further optimisation of these treatments may involve employing the light and atmospheric treatments during film formation to ensure better passivation throughout the bulk of the film. ${ }^{[87]}$ 

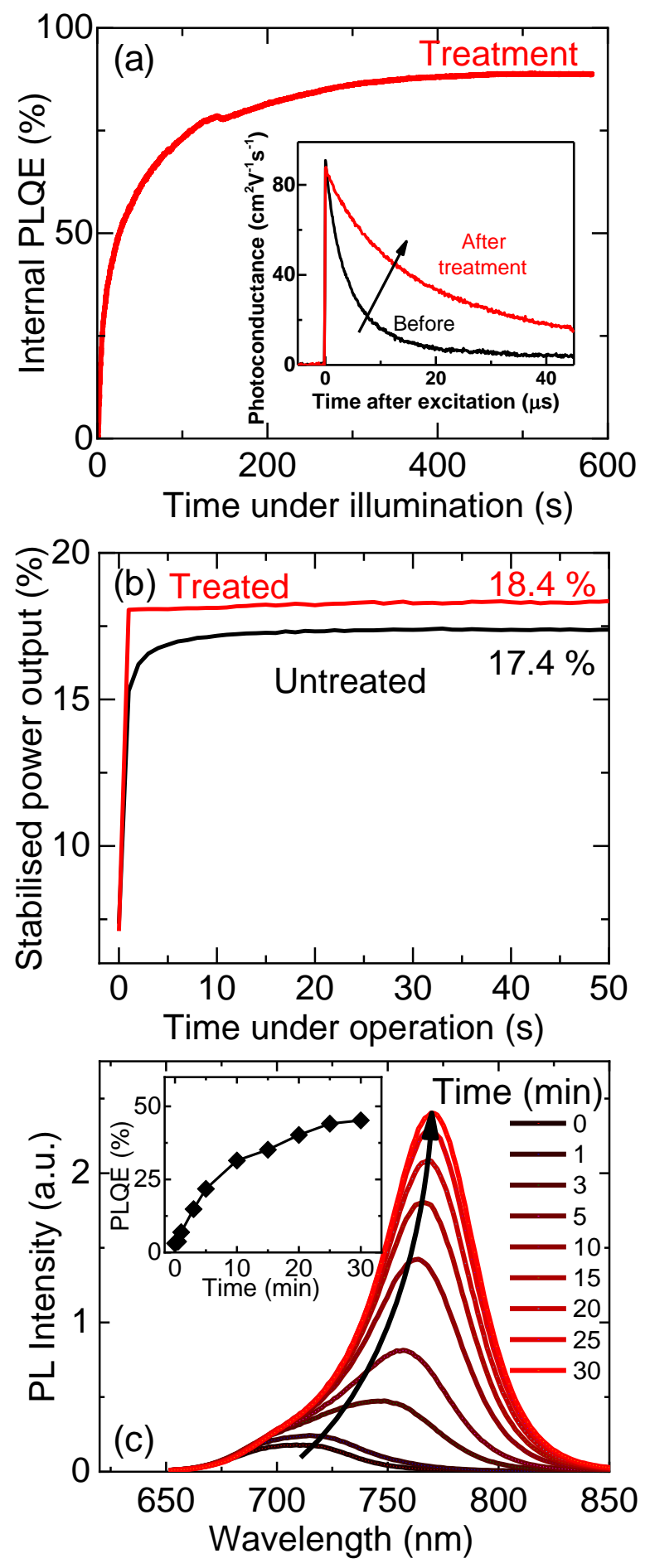

Figure 5. (a) Enhancement in internal PL quantum efficiency (PLQE) of a $\mathrm{MAPbI}_{3}$ thin film using a light and atmospheric treatment (excitation with 532-nm CW at $\sim 150 \mathrm{~mW} / \mathrm{cm}^{2} \sim 45 \%$ relative humidity). ${ }^{[35]}$ (b) Stabilised power output PCE values of solar cell devices comprised of films treated with light and atmospheric exposure (red) and untreated films (black). 
Reproduced under the terms of the Creative Commons CC BY license ${ }^{[35]}$. Copyright 2017, Cell Press. (c) PL spectra of a (FA,MA,Cs) $\mathrm{Pb}\left(\mathrm{I}_{0.6} \mathrm{Br}_{0.4}\right)_{3}$ film over time under 532-nm laser illumination (60 $\mathrm{mW} / \mathrm{cm}^{2}$, ambient laboratory air conditions). The inset is the corresponding external PLQE over time. Reproduced under the terms of the Creative Commons CC BY license. ${ }^{[62]}$ Copyright 2019, Wiley.

Nevertheless, the presence of oxygen and water derivatives (e.g. superoxide), other lightinduced products (e.g. molecular halide species such as $\mathrm{I}_{2}$ ), or strong oxidising agents (e.g. hydrogen peroxide) in the structure of treated samples may not provide sufficient long-term stability required for commercial applications. As one of our colleagues eloquently put it, "It would be like taking an operating flamethrower into your home". ${ }^{[88]}$ Therefore, such treatments involving light and/or atmospheric molecules may not be the most suitable solution for eventual scale up. This motivates the search for alternative species that mimic the positive effect of oxygen species and/or light-induced effects in passivating trap states without the detrimental effects. There are widespread reports of chemical passivation treatments employed either through use of additives in the precursor solutions or through post-treatments of bulk films. These include long-chain 2D molecules that incorporate into the perovskite surface structure and sulphur, nitrogen or phosphorus-containing molecules that interact with surface defects. ${ }^{[89]}$ Use of these molecules to bind to undercoordinated lead may impede the formation of $I_{2}$ at these sites and associated photodarkening processes. ${ }^{[54]}$ One key advantage of the lighttreatment is that charge carriers can be generated in selected regions of the film (dictated, for example, by the excitation wavelength) and can instigate photo-chemical reactions in those specific regions. Furthermore, the selective binding of species such as molecules to defect sites may allow one to direct on an atomic scale where the photo-chemical reactions occur, allowing a more complete amelioration of trap states throughout the bulk of the film than can be achieved 
with chemical surface treatments alone. One could seek to use other small species that could diffuse through the film and selectively bind to defects in a similar way, which also undergo subsequent photochemical reactions as required. Other strategies may involve removing the adsorbed species once the passivation has occurred, provided the passivation does not require the continued presence of the binding species (e.g. if the molecule simply instigates a transformation of that site). In practice, a combination of all of the above may be required to achieve the maximum luminescence yields and film stabilities.

\section{Conclusion}

In this Progress Report, we scrutinised the literature on photobrightening and photodarkening processes in lead halide perovskites, where photobrightening refers to the enhancement in luminescence efficiency through continued light illumination. We highlighted the sensitivity of the photobrightening effect to experimental conditions such as atmosphere, photon energy, photon dose, and perovskite composition and morphology, which ultimately leads to an interplay between photobrightening and photodarkening and the seemingly conflicting reports in the literature. We dissected the literature to summarise the common elements of mechanisms, namely a requirement that there is a source of charge carriers (for example from photoexcitation or strong oxidising agents) to instigate photochemical reactions with atmospheric species and/or ionic species (halides, vacancies or interstitials) to ameliorate defect states. Although there is growing consensus on many aspects, there are a number of outstanding questions that are under debate, particularly related to the nature of the defects being passivated. Finally, the prospects for these treatments for scale-up to push the properties of polycrystalline films towards those of their single-crystal counterparts scaled-up were discussed. We expect that further optimization of these and similar treatments, along with a deeper understanding of underlying mechanisms, will lead to solar and light-emitting device efficiencies approaching their full potential with minimal ionic migration. This may require in- 
situ characterization techniques in which structural, chemical and photophysical properties can be simultaneously probed and interrelated to provide universal mechanisms.

\section{Acknowledgements}

Z.A.-G. acknowledges funding from a Winton Studentship, and ICON Studentship from the Lloyd's Register Foundation. M.A. acknowledges funding from the European Union's Horizon 2020 research and innovation programme under the Marie Skłodowska-Curie grant agreement No 841386. S.D.S acknowledges support from the Royal Society and Tata Group (UF150033). The authors thank the Engineering and Physical Sciences Research Council (EPSRC) for support.

\section{References}

[1] S. D. Stranks, H. J. Snaith, Nat. Nanotechnol. 2015, 10, 391.

[2] M. Saliba, T. Matsui, J.-Y. Seo, K. Domanski, J.-P. Correa-Baena, M. K. Nazeeruddin, S. M. Zakeeruddin, W. Tress, A. Abate, A. Hagfeldt, M. Grätzel, Energy Environ. Sci. 2016, 9, 1989.

[3] W. S. Yang, B.-W. Park, E. H. Jung, N. J. Jeon, Y. C. Kim, D. U. Lee, S. S. Shin, J. Seo, E. K. Kim, J. H. Noh, S. Il Seok, Science 2017, 356, 1376.

[4] G. E. Eperon, M. T. Hörantner, H. J. Snaith, Nat. Rev. Chem. 2017, 1, 0095.

[5] Z.-K. Tan, R. S. Moghaddam, M. L. Lai, P. Docampo, R. Higler, F. Deschler, M. Price, A. Sadhanala, L. M. Pazos, D. Credgington, F. Hanusch, T. Bein, H. J. Snaith, R. H. Friend, Nat. Nanotechnol. 2014, 9, 687.

[6] M. Saliba, T. Matsui, K. Domanski, J.-Y. Seo, A. Ummadisingu, S. M. Zakeeruddin, J.-P. Correa-Baena, W. R. Tress, A. Abate, A. Hagfeldt, M. Gratzel, Science 2016, 354, 206.

[7] D. P. McMeekin, G. Sadoughi, W. Rehman, G. E. Eperon, M. Saliba, M. T. Hörantner, A. Haghighirad, N. Sakai, L. Korte, B. Rech, M. B. Johnston, L. M. Herz, H. J. Snaith, Science 2016, 351, 151.

[8] M. Abdi-Jalebi, M. I. Dar, A. Sadhanala, S. P. Senanayak, M. Franckevičius, N. Arora, Y. Hu, M. K. Nazeeruddin, S. M. Zakeeruddin, M. Grätzel, R. H. Friend, Adv. Energy Mater. 2016, 6, 1502472. 
[9] M. Abdi-Jalebi, Z. Andaji-Garmaroudi, S. Cacovich, C. Stavrakas, B. Philippe, J. M. Richter, M. Alsari, E. P. Booker, E. M. Hutter, A. J. Pearson, S. Lilliu, T. J. Savenije, H. Rensmo, G. Divitini, C. Ducati, R. H. Friend, S. D. Stranks, Nature 2018, 555, 497.

[10] W. Rehman, D. P. McMeekin, J. B. Patel, R. L. Milot, M. B. Johnston, H. J. Snaith, L. M. Herz, Energy Environ. Sci. 2017, 10, 361.

[11] F. Deschler, M. Price, S. Pathak, L. E. Klintberg, D. D. Jarausch, R. Higler, S. Hüttner, T. Leijtens, S. D. Stranks, H. J. Snaith, M. Atatüre, R. T. Phillips, R. H. Friend, J. Phys. Chem. Lett. 2014, 5, 1421.

[12] NREL, "Best Research-Cell Efficiency Chart | Photovoltaic Research | NREL," 2019.

[13] M. A. Green, Y. Hishikawa, E. D. Dunlop, D. H. Levi, J. Hohl-Ebinger, A. W. Y. HoBaillie, Prog. Photovoltaics Res. Appl. 2018, 26, 3.

[14] B. Zhao, S. Bai, V. Kim, R. Lamboll, R. Shivanna, F. Auras, J. M. Richter, L. Yang, L. Dai, M. Alsari, X.-J. She, L. Liang, J. Zhang, S. Lilliu, P. Gao, H. J. Snaith, J. Wang, N. C. Greenham, R. H. Friend, D. Di, Nat. Photonics 2018, 12, 783.

[15] L. M. Pazos-Outón, T. P. Xiao, E. Yablonovitch, J. Phys. Chem. Lett. 2018, 9, 1703.

[16] J.-P. Correa-Baena, M. Saliba, T. Buonassisi, M. Grätzel, A. Abate, W. Tress, A. Hagfeldt, Science 2017, 358, 739.

[17] P. Lopez-Varo, J. A. Jiménez-Tejada, M. García-Rosell, S. Ravishankar, G. GarciaBelmonte, J. Bisquert, O. Almora, Adv. Energy Mater. 2018, 8, 1702772.

[18] O. D. Miller, E. Yablonovitch, S. R. Kurtz, IEEE J. Photovoltaics 2012, 2, 303.

[19] W. Shockley, H. J. Queisser, J. Appl. Phys. 1961, 32, 510.

[20] U. Rau, Phys. Rev. B 2007, 76, 085303.

[21] T. Kirchartz, U. Rau, Adv. Energy Mater. 2018, 8, 1703385.

[22] S. D. Stranks, ACS Energy Lett. 2017, 2, 1515.

[23] J. J. Yoo, S. Wieghold, M. C. Sponseller, M. R. Chua, S. N. Bertram, N. T. P. Hartono, J. S. Tresback, E. C. Hansen, J.-P. Correa-Baena, V. Bulović, T. Buonassisi, S. S. Shin, M. G. Bawendi, Energy Environ. Sci. 2019, 12, 2192.

[24] Z. Liu, L. Krückemeier, B. Krogmeier, B. Klingebiel, J. A. Márquez, S. Levcenko, S. Öz, S. Mathur, U. Rau, T. Unold, T. Kirchartz, ACS Energy Lett. 2019, 4, 110.

[25] S. D. Stranks, R. L. Z. Hoye, D. Di, R. H. Friend, F. Deschler, Adv. Mater. 2018, 1803336.

[26] C. Stavrakas, A. A. Zhumekenov, R. Brenes, M. Abdi-Jalebi, V. Bulović, O. M. Bakr, E. S. Barnard, S. D. Stranks, Energy Environ. Sci. 2018, 11, 2846.

[27] S. D. Stranks, V. M. Burlakov, T. Leijtens, J. M. Ball, A. Goriely, H. J. Snaith, Phys. Rev. Appl. 2014, 2, 034007.

[28] T. Leijtens, G. E. Eperon, A. J. Barker, G. Grancini, W. Zhang, J. M. Ball, A. R. S. 
Kandada, H. J. Snaith, A. Petrozza, Energy Environ. Sci. 2016, 9, 3472.

[29] G.-J. A. H. Wetzelaer, M. Scheepers, A. M. Sempere, C. Momblona, J. Ávila, H. J. Bolink, Adv. Mater. 2015, 27, 1837.

[30] D. Shi, V. Adinolfi, R. Comin, M. Yuan, E. Alarousu, A. Buin, Y. Chen, S. Hoogland, A. Rothenberger, K. Katsiev, Y. Losovyj, X. Zhang, P. A. Dowben, O. F. Mohammed, E. H. Sargent, O. M. Bakr, Science 2015, 347, 519.

[31] T. M. Brenner, D. A. Egger, L. Kronik, G. Hodes, D. Cahen, Nat. Rev. Mater. 2016, 1, 15007.

[32] Z. Chen, B. Turedi, A. Y. Alsalloum, C. Yang, X. Zheng, I. Gereige, A. AlSaggaf, O. F. Mohammed, O. M. Bakr, ACS Energy Lett. 2019, 4, 1258.

[33] J. F. Galisteo-López, M. Anaya, M. E. Calvo, H. Míguez, J. Phys. Chem. Lett. 2015, 6, 2200 .

[34] Y. Tian, M. Peter, E. Unger, M. Abdellah, K. Zheng, T. Pullerits, A. Yartsev, V. Sundström, I. G. Scheblykin, Phys. Chem. Chem. Phys. 2015, 17, 24978.

[35] R. Brenes, D. Guo, A. Osherov, N. K. Noel, C. Eames, E. M. Hutter, S. K. Pathak, F. Niroui, R. H. Friend, M. S. Islam, H. J. Snaith, V. Bulović, T. J. Savenije, S. D. Stranks, Joule 2017, 1, 155.

[36] M. Anaya, J. F. Galisteo-López, M. E. Calvo, J. P. Espinós, H. Míguez, J. Phys. Chem. Lett. 2018, 9, 3891.

[37] A. Senocrate, T. Acartürk, G. Y. Kim, R. Merkle, U. Starke, M. Grätzel, J. Maier, J. Mater. Chem. A 2018, 6, 10847.

[38] D. W. deQuilettes, W. Zhang, V. M. Burlakov, D. J. Graham, T. Leijtens, A. Osherov, V. Bulović, H. J. Snaith, D. S. Ginger, S. D. Stranks, Nat. Commun. 2016, 7, 11683.

[39] Y. Yamada, M. Endo, A. Wakamiya, Y. Kanemitsu, J. Phys. Chem. Lett. 2015, 6, 482.

[40] R. Gottesman, L. Gouda, B. S. Kalanoor, E. Haltzi, S. Tirosh, E. Rosh-Hodesh, Y. Tischler, A. Zaban, C. Quarti, E. Mosconi, F. De Angelis, J. Phys. Chem. Lett. 2015, 6, 2332.

[41] S. D. Stranks, A. Petrozza, Nat. Photonics 2016, 10, 562.

[42] D. W. deQuilettes, K. Frohna, D. Emin, T. Kirchartz, V. Bulovic, D. S. Ginger, S. D. Stranks, Chem. Rev. 2019, 119, 11007.

[43] B. Chen, M. G. Kanatzidis, O. Durand, J.-C. Blancon, R. Verduzco, J. Even, J. W. Strzalka, W. Nie, S. Tretiak, R. Asadpour, P. M. Ajayan, M. A. Alam, A. D. Mohite, H. Tsai, C. C. Stoumpos, Science 2018, 360, 67.

[44] E. Mosconi, D. Meggiolaro, H. J. Snaith, S. D. Stranks, F. De Angelis, Energy Environ. Sci. 2016, 9, 3180.

[45] Y. Tian, A. Merdasa, E. Unger, M. Abdellah, K. Zheng, S. McKibbin, A. Mikkelsen, T. Pullerits, A. Yartsev, V. Sundström, I. G. Scheblykin, J. Phys. Chem. Lett. 2015, 6, 4171. 
[46] H.-H. Fang, S. Adjokatse, H. Wei, J. Yang, G. R. Blake, J. Huang, J. Even, M. A. Loi, Sci. Adv. 2016, 2, e1600534.

[47] R. Brenes, C. Eames, V. Bulović, M. S. Islam, S. D. Stranks, Adv. Mater. 2018, 30, 1706208.

[48] W.-A. Quitsch, D. W. DeQuilettes, O. Pfingsten, A. Schmitz, S. Ognjanovic, S. Jariwala, S. Koch, M. Winterer, D. S. Ginger, G. Bacher, J. Phys. Chem. Lett. 2018, 9, 2062.

[49] W. Rehman, R. L. Milot, G. E. Eperon, C. Wehrenfennig, J. L. Boland, H. J. Snaith, M. B. Johnston, L. M. Herz, Adv. Mater. 2015, 27, 7938.

[50] D. Guo, Z. Andaji Garmaroudi, M. Abdi-Jalebi, S. D. Stranks, T. J. Savenije, ACS Energy Lett. 2019, 2360.

[51] Y. Wang, Y. Ren, S. Zhang, J. Wu, J. Song, X. Li, J. Xu, C. H. Sow, H. Zeng, H. Sun, Commun. Phys. 2018, 1, 96.

[52] S. G. Motti, M. Gandini, A. J. Barker, J. M. Ball, A. R. Srimath Kandada, A. Petrozza, ACS Energy Lett. 2016, $1,726$.

[53] N. Aristidou, C. Eames, I. Sanchez-Molina, X. Bu, J. Kosco, M. S. Islam, S. A. Haque, Nat. Commun. 2017, 8, 15218.

[54] S. G. Motti, D. Meggiolaro, A. J. Barker, E. Mosconi, C. A. R. Perini, J. M. Ball, M. Gandini, M. Kim, F. De Angelis, A. Petrozza, Nat. Photonics 2019, 13, 532.

[55] C. M. Sutter-Fella, Y. Li, M. Amani, J. W. Ager, F. M. Toma, E. Yablonovitch, I. D. Sharp, A. Javey, Nano Lett. 2016, 16, 800.

[56] A. Merdasa, A. Kiligaridis, C. Rehermann, M. Abdi-Jalebi, J. Stöber, B. Louis, M. Gerhard, S. D. Stranks, E. L. Unger, I. G. Scheblykin, ACS Energy Lett. 2019, 4, 1370.

[57] M. Gerhard, B. Louis, R. Camacho, A. Merdasa, J. Li, A. Kiligaridis, A. Dobrovolsky, J. Hofkens, I. G. Scheblykin, Nat. Commun. 2019, 10, 1698.

[58] A. Merdasa, Y. Tian, R. Camacho, A. Dobrovolsky, E. Debroye, E. L. Unger, J. Hofkens, V. Sundström, I. G. Scheblykin, ACS Nano 2017, 11, 5391.

[59] A. Halder, N. Pathoor, A. Chowdhury, S. K. Sarkar, J. Phys. Chem. C 2018, 122, 15133.

[60] J. F. Galisteo-López, M. E. Calvo, T. C. Rojas, H. Míguez, ACS Appl. Mater. Interfaces 2019, 11, 6344.

[61] E. T. Hoke, D. J. Slotcavage, E. R. Dohner, A. R. Bowring, H. I. Karunadasa, M. D. McGehee, Chem. Sci. 2015, 6, 613.

[62] Z. Andaji-Garmaroudi, M. Abdi-Jalebi, D. Guo, S. Macpherson, A. Sadhanala, E. M. Tennyson, E. Ruggeri, M. Anaya, K. Galkowski, R. Shivanna, K. Lohmann, K. Frohna, S. Mackowski, T. J. Savenije, R. H. Friend, S. D. Stranks, Adv. Mater. 2019, 31,1902374 .

[63] D. J. Slotcavage, H. I. Karunadasa, M. D. McGehee, ACS Energy Lett. 2016, 1, 1199. 
[64] S. J. Yoon, S. Draguta, J. S. Manser, O. Sharia, W. F. Schneider, M. Kuno, P. V. Kamat, ACS Energy Lett. 2016, 1, 290.

[65] P. Fassl, V. Lami, A. Bausch, Z. Wang, M. T. Klug, H. J. Snaith, Y. Vaynzof, Energy Environ. Sci. 2018, 11, 3380.

[66] R. E. Beal, D. J. Slotcavage, T. Leijtens, A. R. Bowring, R. A. Belisle, W. H. Nguyen, G. F. Burkhard, E. T. Hoke, M. D. McGehee, J. Phys. Chem. Lett. 2016, 7, 746.

[67] D. B. F. D. S. Feldmann, S. Macpherson, S. P. Senanayak, M. Abdi-Jalebi, J. P.H. Rivett, G. Nan, G. D. Tainter, T. A. S. Doherty, K. Frohna, E. Ringe, R. H. Friend, H. Sirringhaus, M. Saliba, Nat. Photonics 2019, In Press.

[68] T. Leijtens, S. D. Stranks, G. E. Eperon, R. Lindblad, E. M. J. Johansson, I. J. McPherson, H. Rensmo, J. M. Ball, M. M. Lee, H. J. Snaith, ACS Nano 2014, 8, 7147.

[69] C. Eames, J. M. Frost, P. R. F. Barnes, B. C. O’Regan, A. Walsh, M. S. Islam, Nat. Commun. 2015, 6, 7497.

[70] A. Abrusci, S. D. Stranks, P. Docampo, H.-L. Yip, A. K.-Y. Jen, H. J. Snaith, Nano Lett. 2013, 13, 3124.

[71] J. S. W. Godding, A. J. Ramadan, Y.-H. Lin, K. Schutt, H. J. Snaith, B. Wenger, Joule 2019, DOI 10.1016/j.joule.2019.08.006.

[72] A. Osherov, E. M. Hutter, K. Galkowski, R. Brenes, D. K. Maude, R. J. Nicholas, P. Plochocka, V. Bulović, T. J. Savenije, S. D. Stranks, Adv. Mater. 2016, 28, 10757.

[73] D. Meggiolaro, E. Mosconi, F. De Angelis, ACS Energy Lett. 2017, 2, 2794.

[74] J. H. Noh, S. H. Im, J. H. Heo, T. N. Mandal, S. Il Seok, Nano Lett. 2013, 13, 1764.

[75] A. M. A. Leguy, Y. Hu, M. Campoy-Quiles, M. I. Alonso, O. J. Weber, P. Azarhoosh, M. van Schilfgaarde, M. T. Weller, T. Bein, J. Nelson, P. Docampo, P. R. F. Barnes, Chem. Mater. 2015, 27, 3397.

[76] C. Müller, T. Glaser, M. Plogmeyer, M. Sendner, S. Döring, A. A. Bakulin, C. Brzuska, R. Scheer, M. S. Pshenichnikov, W. Kowalsky, A. Pucci, R. Lovrinčić, Chem. Mater. 2015, 27, 7835.

[77] T. W. Jones, A. Osherov, M. Alsari, M. Sponseller, B. C. Duck, Y. K. Jung, C. Settens, F. Niroui, R. Brenes, C. V. Stan, Y. Li, M. Abdi-Jalebi, N. Tamura, J. E. MacDonald, M. Burghammer, R. H. Friend, V. Bulović, A. Walsh, G. J. Wilson, S. Lilliu, S. D. Stranks, Energy Environ. Sci. 2019, 12, 596.

[78] J.-S. Park, J. Calbo, Y.-K. Jung, L. D. Whalley, A. Walsh, ACS Energy Lett. 2019, 4, 1321.

[79] J. Zhao, Y. Deng, H. Wei, X. Zheng, Z. Yu, Y. Shao, J. E. Shield, J. Huang, Sci. Adv. 2017, 3, eaao5616.

[80] N. Rolston, K. A. Bush, A. D. Printz, A. Gold-Parker, Y. Ding, M. F. Toney, M. D. McGehee, R. H. Dauskardt, Adv. Energy Mater. 2018, 8, 1802139.

[81] X.-Y. Zhu, V. Podzorov, J. Phys. Chem. Lett. 2015, 6, 4758. 
[82] D. W. deQuilettes, S. Koch, S. Burke, R. K. Paranji, A. J. Shropshire, M. E. Ziffer, D. S. Ginger, ACS Energy Lett. 2016, 1, 438.

[83] I. L. Braly, D. W. DeQuilettes, L. M. Pazos-Outón, S. Burke, M. E. Ziffer, D. S. Ginger, H. W. Hillhouse, Nat. Photonics 2018, 12, 355.

[84] E. Yablonovitch, D. L. Allara, C. C. Chang, T. Gmitter, T. B. Bright, Phys. Rev. Lett. 1986, 57, 249.

[85] P. Saint-Cast, D. Kania, M. Hofmann, J. Benick, J. Rentsch, R. Preu, Appl. Phys. Lett. 2009, 95, 151502.

[86] A. G. Aberle, Prog. Photovoltaics Res. Appl. 2000, 8, 473.

[87] A. Ummadisingu, L. Steier, J.-Y. Seo, T. Matsui, A. Abate, W. Tress, M. Grätzel, Nature 2017, 545, 208.

[88] Hernan Miguez, personal communications.

[89] N. K. Noel, S. D. Stranks, A. Abate, C. Wehrenfennig, S. Guarnera, A.-A. Haghighirad, A. Sadhanala, G. E. Eperon, S. K. Pathak, M. B. Johnston, A. Petrozza, L. M. Herz, H. J. Snaith, Energy Environ. Sci. 2014, 7, 3061. 